\title{
Tobacco blindness
}

\author{
Stephen J Jay
}

Tobacco "blindness" or amblyopia has been recognised clinically for more than 150 years. ${ }^{1}$ The "blind spot" for tobacco in scholarly publications has received scant attention, however, and is a current and pressing concern.

How does this amblyopia for tobacco manifest itself? First, publications of original research often fail to measure and report the use of tobacco products and exposure to environmental tobacco smoke (ETS) by study subjects, even when research objectives and outcomes measurements are likely to be affected by smoking, the use of smokeless tobacco, or exposure to ETS. Second, review articles in widely read biomedical journals often fail to acknowledge the importance of tobacco use and exposure to ETS when the subject under discussion would warrant it. And third, national consensus conference statements and practice guidelines may inappropriately omit discussion and recommendations regarding tobacco use and ETS.

Some recent examples which have come to my attention are offered as anecdotal yet compelling evidence of the existence of "tobacco blindness" among our colleagues and "experts" in the biomedical sciences.

\section{Scientific studies}

Two long-term studies of asthma morbidity and mortality published in the Nerw England fournal of Medicine failed to quantify smoking status of subjects adequately; one study provided no data regarding smoking rates and neither study included data on ETS or even recognised it as potentially related to clinical outcomes, despite enormous evidence, published widely over many years, relating asthma to smoking and ETS.

The authors of a recent evaluation of adverse outcomes of underuse of $\beta$-blockers in elderly survivors of acute myocardial infarction indicated that a limitation of their research was their inability to measure lifestyle factors, including smoking, that were not contained in the administrative databases that were used for analyses in this study. ${ }^{3}$ The importance of controlling for both active and passive smoking in this and other investigations of pharmacological agents on measures of cardiovascular function and outcomes was noted. ${ }^{4}$ Finally, in a clinical investigation of the use of the six-minute walk test in the evaluation of functional capacity and survival in severe heart failure, ${ }^{5}$ no data were presented nor mention made of the potential confounding effects on study results of exposure to ETS. ${ }^{6}$

\section{Review articles}

In a recent review of the pharmacological and non-pharmacological management of chronic heart failure (HF) published in a prestigious medical journal, ${ }^{7}$ the author did not mention the role of active or passive smoking in the pathogenesis of $\mathrm{HF}$ or the potential benefits of smoking cessation (active or passive) in the management of these patients. Since the 1960 s numerous studies have been published in peerreviewed biomedical journals regarding the adverse effects of cigarette smoke, inhaled lowconcentration carbon monoxide, and ETS on cardiovascular pathophysiology, morbidity, and mortality. ${ }^{8}$

\section{Consensus conferences/practice guidelines}

In two widely circulated consensus conference clinical guidelines for primary-care physicians regarding the management of $\mathrm{HF}$, the importance of smoking cessation and avoidance of ETS or other sources of environmental carbon monoxide were not mentioned. ${ }^{9}{ }^{10}$ These guidelines were published under the auspices of the Agency for Health Care Policy and Research (US Department of Health and Human Services) and the Rand Corporation (1994), and a joint task force of the American College of Cardiology and the American Heart Association (1995).

A recent National Institutes of Health consensus development conference on the prevention of cervical cancer ${ }^{11}$ provided no data nor recommendations regarding the relationship between cervical cancer and smoking or exposure to ETS; numerous scientific publications over approximately 15 years have documented the relation of smoking, or exposure to ETS, or both, to the prevalence of cervical dysplasia, carcinoma in situ, and cervical cancer. ${ }^{12}$ I raised concerns regarding these deficiencies in this otherwise excellent NIH consensus development conference statement on 13 September 1996, 6 February 1997, and 20 March 1997. A communication was received from the NIH Office of Medical Applications of Research (28 March 1997) that acknowledged the problem but indicated that "since we didn't have any data regarding smoking as a risk factor ... they could [not] have put it in the statement". It is not clear 
whether the NIH consensus development process can or will allow the cervical cancer statement to be amended to include information on tobacco. I am seeking clarification on this point.

The problems that these examples raise seem to reside in three general areas.

- The lack of appropriate data available to researchers in medical records and computer databases regarding smoking, the use of smokeless tobacco, and exposure to ETS.

- The failure of researchers to incorporate available methodology for quantifying tobacco use and ETS exposure into research design and protocols for grant proposals and research publications.

- The failure of editors and reviewers to challenge authors to address tobacco use and ETS exposure, where appropriate, in scientific studies, clinical review articles, and consensus conference statements and publications of clinical guidelines.

Editors, journal article expert reviewers, and researchers would likely benefit from the publication of state-of-the-art guidelines regarding the appropriate methodology for quantifying tobacco use and exposure to ETS for use in prospective research trials. Such information could provide needed guidelines that would be useful to reviewers in judging the quality of research manuscripts and grant proposals; to researchers attempting to answer questions that could be confounded by inadequate control for tobacco use; and to ipvestigators involved in meta-analyses or systematic reviews where explicit criteria regarding research objectives and outcomes measures are central to this type of investigation.

Until more thought and study ar these issues, we will likely continue to see evidence of "tobacco blindness" in the biomedical literature.

1 Kellogg JH. Tobaccoism or how tobacco kills Battle Creek, Michigan: Modern Medicine Publishing, 1922:91.

2 Jay SJ. Patterns of mortality from asthma (IRter to the editor). $N$ Engl F Med 1995;332:1379-81.

3 Soumerai SB, McLaughlin TJ, Spiegelman $\overrightarrow{D_{3}}$ et al. Adverse outcomes of underuse of $\beta$-blockers in eldeply survivors of acute myocardial infarction. $¥ A M A$ 1996; 7 7 7:115-21.

4 Jay SJ. Adverse outcomes of underuse o $\beta$-blockers in elderly survivors of acute myocardial infacotion (letter to the editor). $F A M A$ 1997;277:1434-5.

5 Cahalin LP, Mathier, MA, Semigran M et al. The Six-Minute Walk Test predicts peak oxygen, uptake and survival in patients with advanced hears failure. Chest 1996;110:325-32.

6 Jay SJ. Passive smoking and the Six-Minutg Walk Test in heart failure (letter to the editor). Chest 1987;112:289-90. 7 Cohn JN. The management of chronic hearffailure. $N$ Engl f Med 1996;335:490-8.

8 Glantz SA, Parmley WW. Passive smol disease. Mechanisms and risk. $¥ A M A$ 199:273:1047-53.

9 Guidelines for the evaluation and management of heart failure. American College of Cardiology/American Heart Association Task Force on Practice Guidenes (Committee on Evaluation and Management of Heart Failure). Circulation 1995;92:2764-83.

10 Konstam MA, Dracup K, Baker DW, et a $\mathrm{P}$ Heart failure: evaluation and care of patients with left ventricular systolic dysfunction. $\mathcal{F}$ Cardiac Failure 1995;1:183긍.

11 Cervical Cancer. NIH Consensus Statement 1996 April 1-3; 14(1):1-38.

12 Slattery ML, Robison LM, Schuman KL, $\mathbb{\Phi}_{t}$ al. Cigarette smoking and exposure to passive smoke aretrisk factors for cervical cancer. $\mathscr{F} A M A$ 1989;261:1593-8. 윽 